\title{
Subempleo laboral en las industrias productivas de Nicaragua
}

\author{
Francisco Mendoza Tijerino* y Álvaro Altamirano Montoya ${ }^{* *}$
}

\section{Recibido: junio de 2012 / Aceptado: febrero de 2013}

En el presente artículo se analiza el subempleo utilizando información de la última Encuesta Continua de Hogares 2010, publicada por el Instituto Nacional de Información del Desarrollo (INIDE) de Nicaragua. Se busca identificar las industrias que presenten más problemas de subempleo, tomando en cuenta que los individuos que se encuentren subempleados son individuos que están inconformes e insatisfechos al momento de realizar las labores productivas. Se encuentra que, en relación a las demás industrias del país, la industria de la construcción es la que presenta mayores probabilidades de contar con empleados con deseos de trabajar más y no poder hacerlo.

Palabras clave: subempleo / industrias productivas / Nicaragua

\section{Introducción}

Según la Encuesta de Medición del Nivel de Vida (EMNV) para el año 2009, elaborada por el Instituto Nacional de Información del Desarrollo (INIDE, 2010), el 56 por ciento de los hogares encuestados ha indicado que el principal problema que percibe Nicaragua y que afecta la seguridad ciudadana es el desempleo. Cabe resaltar que la tasa de desempleo promedio oficial ha sido de 6.35 por ciento de la Población Económicamente Activa (PEA) desde el año 2003 hasta el año 2009, una tasa de desempleo que se considera baja si se compara con las tasas de desempleo de otros países latinoamericanos.

* Estudiante de Economía Aplicada en la Universidad Centroamericana. Rotonda Rubén Dario 150 mts. al oeste. Apartado 69, Managua, Nicaragua. Correo electrónico:franciscomt91@hotmail.com

**Departamento de Economía Aplicada de laUniversidad Centroamericana. Rotonda Rubén Dario 150 mts. al oeste. Apartado 69, Managua, Nicaragua. Correo electrónico: unilyrics@hotmail.com 
Dada esta discrepancia en percepciones, se presenta una paradoja que puede ser aclarada si se analiza otro indicador que capture más a cabalidad la problemática que aprecia la ciudadanía. Este indicador es el subempleo, un concepto utilizado para caracterizar una situación en la que la fuerza de trabajo permanece inactiva o poco aprovechada -y también infra-remunerada-, ya sea por insuficiencia de capital $\mathrm{u}$ otros factores, pero que es imprescindible toda ella en determinados momentos del ciclo productivo (Ahijado, 1985).

La dificultad que plantea el subempleo radica en que se desperdicia potencial productivo de los trabajadores y esta situación ha envuelto a un gran segmento de la fuerza laboral nicaragüense en los últimos años. De igual forma, no tener una homogeneidad en dicho potencial de producción en las distintas industrias dificulta conocer el peso que puede tener el factor trabajo en los procesos productivos.

Esta contribución busca contribuir a llenar el vacío existente en el análisis sobre el subempleo en Nicaragua. Es escaza la literatura concerniente a la temática a pesar de ser una problemática de envergadura. El artículo persigue identificar cuáles son las industrias productivas de Nicaragua que tienen mayores probabilidades de presentar agentes económicos bajo subempleo. El subempleo se analiza como inconformidad en las industrias. Se habla de inconformidad ya que el empleado desea trabajar más horas de las que el mercado le permite.

La importancia de la distinción e identificación a nivel industrial es que puede plantear el inicio de acciones de política económica, es decir, sugerir hacia dónde se deben dirigir los esfuerzos de las políticas de empleo y, por ende, las bases o los criterios de dichas políticas.

La herramienta a utilizar fue la aplicación de un modelo econométrico con respuesta cualitativa, en donde el valor de 1 se asignó al agente económico que desea trabajar más horas y no puede y 0 al agente económico que no lo desea. Se encontró la probabilidad de estar subempleado dado que el agente económico pertenece a $\chi_{i}$ industria.

Las industrias a utilizar fueron estimadas a partir de la distinción establecida en el manual publicado en el Banco Central de Nicaragua (BCN) titulado "Sistema de Cuentas Nacionales de Nicaragua año 1994”, sin embargo no es una distinción definitiva para propósitos de estimación econométrica.

Este artículo se divide en seis secciones principales. En la sección 2 se indican los antecedentes del mercado laboral de Nicaragua; en la sección 3 se presenta la literatura referente a la temática; seguidamente, en la sección 4 , los materiales y el método a utilizar; en la sección 5 se establece el marco empírico, en el cual, inicialmente se caracterizará críticamente el subempleo en el país a nivel macroeconómico y a nivel microeconómico para luego proceder a la estimación econométrica; posteriormente, en la sección 6 se incluirán las conclusiones al respecto.

\section{Antecedentes}

El entramado institucional, en el sentido de North (1990) - es decir, todas las reglas y convenciones sociales, formales e informales - es de gran importancia para analizar el mercado de factores de producción y, más específicamente, el subempleo en el mercado de trabajo nicaragüense. 
Las primeras afectaciones o cambios en materia de legislación laboral, a raíz de los programas de ajuste estructural y del cambio hacia un modelo abierto, se dieron en 1994 al modificarse algunas de las competencias del Ministerio del Trabajo que minaron su capacidad de defender a los trabajadores. Estas competencias se referían a la autoridad que tenía el Ministerio del Trabajo para resolver reclamos de los trabajadores de hasta un máximo de 5,000 córdobas, relacionados con prestaciones laborales.

- En 1996 se reformó el Código del Trabajo con normativas de flexibilización laboral, entre las cuales se destacan, por importancia:

- La posibilidad de contratar a un trabajador mediante contratos de plazo determinado, sin mínimos ni máximos de tiempo.

- La facultad de suspender labores por falta de materia prima o por motivos económicos o técnicos.

- Se limita la indemnización por despido injustificado a un mes de salario por cada uno de los tres primeros años trabajados y a 20 días por los siguientes, con un tope máximo de cinco meses de salario.

Gamboa (2007) considera que la mayor debilidad del entorno legislativo laboral de Nicaragua surge de la poca aplicabilidad del Código del Trabajo. Dentro de las omisiones prácticas más importantes de la ley encontramos la aplicación correcta del salario mínimo, de la estabilidad laboral, de la salud ocupacional, de la seguridad social, entre otros.

Sumado a esto, el pobre conocimiento de los trabajadores sobre sus derechos laborales y los medios para defenderlos, es una limitante para el fortalecimiento de las condiciones del mercado laboral nicaragüense.

Esta falta de práctica se debe principalmente a la escasez de recursos y capacidades del Ministerio del Trabajo (MITRAB) para vigilar los derechos de los trabajadores a través de inspecciones rutinarias. No obstante, el MITRAB ha realizado esfuerzos desde 2005 para ampliar la cobertura en dicho aspecto.

\section{Revisión de literatura}

La literatura concerniente al subempleo laboral, a pesar de tener un enfoque de aplicación distinto al que se busca en el presente documento, resalta rasgos claves de los agentes económicos inmersos en tales situaciones. Las características que presenten los trabajadores a nivel general en una economía pueden servir de guía para lograr una caracterización más delimitada a nivel de industrias.

Bell y Blanchflower (2011) establecen un estudio del subempleo laboral en un momento de choques de demanda para la economía, comprendido en el Reino Unido. La investigación construye un nuevo análisis para diferentes edades de los agentes económicos, considerando un marco conceptual en el que los costos son los que previenen un correcto ajuste al equilibrio de manera rápida. El marco de referencia en el que se basa el estudio establece que, tras algún choque de demanda, las empresas ajustarán inicialmente la utilización del factor trabajo antes del stock de trabajo con el que cuenta. La premisa que sostiene el marco de referencia utilizado radica en que es más costoso para una firma despedir trabajadores y contratar nuevos 
(conlleva costos de entrenamiento, despido, contrato), por lo que el ajuste conlleva a una búsqueda de mayores contratos parciales por parte de los empleadores, generando así empleados con deseos de trabajar más y con más ganas de ejercer su potencial.

Así mismo, el ajuste de una empresa con respecto al factor trabajo también se puede llevar a cabo tomando en cuenta el tiempo de laborar que tiene un individuo en dicha empresa. Si el empleado presenta mayor tiempo trabajando en la firma es de esperarse que la firma reduzca sus horas de trabajo en lugar de despedirlo, asumiendo siempre que en la economía hay un choque de demanda.

En el contexto nicaragüense, una aplicación de tal marco referencial sería inviable ya que descansa en el hecho de contar con trabajadores (al menos en su mayoría) formales, donde al momento del despido y el contrato existan suficientes trabas legales que impidan minimizar costos de ajuste. Tanto tener que pagar por prestaciones al despedir, como tener que entrenar a un nuevo empleado, se perfilan como obstáculos al ajuste.

Nicaragua cuenta con niveles de formalidad muy bajos en comparación a los niveles de informalidad que imperan en la economía, lo que invalida que los costos de despido y de contrato conlleven altos costos para las empresas. Durante el período 2003-2009 el empleo formal en la economía nicaragüense ha representado alrededor del 35\% de la PEA ocupada.

Por otro lado, Bernal (2008), en su estudio sobre la informalidad laboral en Colombia, concibe al subempleo como una medida de satisfacción por parte del trabajador. De esto se infiere que querer trabajar más y no poder es evidencia de trabajadores no satisfechos. Trabajar en condiciones de inconformidad implica que no se logra potenciar la capacidad productiva del empleado.

La autora, en su estudio realiza una conexión muy clara entre el subempleo y la informalidad: los trabajadores que se encuentran laborando informalmente son más probables de reportar deseos de trabajar más horas de las que trabajan ordinariamente. Si se toma en cuenta que en Nicaragua 7 de cada 10 trabajadores son informales, es de esperarse que un gran segmento de la población presente pérdidas de satisfacción al trabajar.

La condición antes expuesta realza la preocupación pues, según el BCN, el conjunto de industrias agropecuarias es el que logra abarcar a la mayor parte de la fuerza laboral en Nicaragua. Es éste, precisamente, el grupo que presenta mayor informalidad debido a que la cobertura del sistema de seguridad social en las áreas rurales es muy inferior a la cobertura en el área urbana pues en el país no existe un régimen de seguridad social para los trabajadores del campo.

\section{Materiales y método}

\subsection{Encuesta}

Se hizo uso de la Encuesta Continua de Hogares del año 2010 (en adelante, ECH) proporcionada por el INIDE (2010) como instrumento base para la elaboración del artículo. La encuesta cuenta con 31,947 observaciones (promedio de los cuatro trimestres del año 2010) y fue realizada en todos los departamentos de Nicaragua. 
Dicha encuesta cuenta, en promedio, con el 42.5 por ciento de su muestra recogida en áreas rurales, y el restante 56.5 por ciento en el área urbana.

Cabe señalar que la ECH cuenta con cuatro períodos, recogiéndose información en cada uno de los cuatro trimestres. La aplicación del método econométrico que se presentará posteriormente se realizó para los cuatro periodos que la encuesta permite diferenciar.

Con base en el instrumento se distingue entre los trabajadores de 15 industrias productivas del país: industrias agrícolas, industrias pecuarias, industrias de servicios personales y empresariales, industrias forestales, industrias pesqueras, industrias de explotación minera, industrias de electricidad y agua, industria comercial, hotelera y de restaurantes, industrias de transporte y comunicaciones, industrias de servicios de intermediación financiera, industria de la construcción e industrias de servicios públicos.

El criterio para agrupar las industrias se fundamenta en la definición presentada en el Sistema de Cuentas Nacionales de 1993, publicado por el Banco Central de Nicaragua, en el cual se indica que: "La industria se define como un conjunto de establecimientos dedicados a la misma actividad económica" (BCN, 1994, p. 13).

\subsection{Metodología}

Se utilizó un modelo econométrico con respuesta cualitativa (variable dicótoma). Los tres modelos de respuesta binaria más utilizados son el Modelo Lineal Probabilístico (MLP), el Modelo Probit y el Modelo Logit.

En el presente trabajo se utiliza el modelo Logit, en detrimento del uso del modelo lineal de probabilidad, ya que éste tiene ciertas limitaciones al presentar probabilidades que superan la unidad y el cero en los efectos parciales en alguna de las variables explicativas.

Para la estimación de los modelos Logit con respuesta cualitativa 1 o 0 se hace uso de la técnica de Máxima Verosimilitud y se asume que la probabilidad de ocurrencia de un evento viene dada por la función:

$$
\text { (1) } \quad p=F(Z)=\frac{1}{1+e^{-Z}}
$$

En el modelo Logit se supera la dificultad presentada en el MLP ya que mientras $Z$ tiende a infinito, $\mathrm{e}^{\mathrm{Z}}$ tiende a 0 y $\mathrm{p}$ tiene un límite superior de 1.

Donde $\mathrm{Z}=\alpha+\delta \mathrm{iXj}$

Así mismo, mientras $\mathrm{Z}$ tiende a menos infinito, $\mathrm{e}^{-\mathrm{Z}}$ tiende a infinito y $\mathrm{p}$ tiene un límite inferior de cero.

En un caso específico, el modelo logístico quedará representado por la forma:

$$
\text { (2) } \quad p=\frac{1}{1+e^{-\alpha-\delta i \boldsymbol{j} j}}
$$


Donde $\delta \mathrm{i}$ representa los parámetros a estimar y $\boldsymbol{X}_{\mathrm{j}}$ representa todas las variables explicativas a los resultados 1 y 0 que toma la variable dependiente " $Y$ "

\section{Marco empírico}

\subsection{Contexto macroeconómico}

La economía de Nicaragua se clasifica como una economía pequeña y abierta. Pequeña porque contribuye con apenas el 5 por ciento a la producción total del istmo centroamericano. Abierta porque la suma de sus importaciones y exportaciones representa para el año 2011 más del 100 por ciento de su producción anual.

En este sentido, se tiene una actividad económica muy vulnerable a shocks económicos externos, principalmente - por el lado de la oferta - a alzas en el precio internacional del petróleo y - por el lado de la demanda - a recesiones que sufran nuestros principales socios comerciales. El ejemplo más reciente de esta vulnerabilidad fue la recesión de 2009, que se propagó en Nicaragua por las vías del crecimiento de la producción, el subempleo, el comercio exterior, los ingresos tributarios, las remesas familiares y la inversión extranjera directa. Nicaragua entró en recesión desde el segundo trimestre de 2009 y salió técnicamente de esa recesión durante el segundo trimestre de 2010. ${ }^{1}$

Desde otro enfoque, Nicaragua es también (aún) un país pobre y muy endeudado en términos de producción porque, según la EMNV 2009 (INIDE, 2009), el $42.5 \%$ de la población es pobre y porque el saldo de la deuda pública total (externa e interna) representó en 2011 el 72\% del PIB de ese mismo año, aunque en la actualidad sólo se honra el 60\% del saldo "contable" de la deuda porque el 40\% aún se encuentra pendiente de reestructuración en el marco de la Iniciativa de Países Pobres Muy Endeudados (PPME) .

De acuerdo con datos del BCN (2012), en 2011 el 77.2\% de las personas ocupadas laboraron en establecimientos que no poseían registros contables. Esto quiere decir que 2 millones 167 mil personas no cotizan seguridad social ni pagan impuestos sobre la renta. Por otro lado, la tasa de subempleo (asociada con la informalidad) fue del $49.1 \%$ de la PEA, es decir que 1 millón 470 mil personas no laboran la jornada legal de ocho horas diarias y, si las laboran, devengan un salario inferior al salario mínimo legal. En resumen, el 55.4\% de la PEA tiene problemas de empleo, o sea que existe 1 millón 660 mil personas desempleadas en forma abierta y subempleadas. Todo esto ilustra cómo la "microeconomía" no está bien, a pesar de que Nicaragua sea "el mejor alumno" del Fondo Monetario Internacional en el ámbito macroeconómico.

Lo anterior indica que el mercado laboral de Nicaragua muestra una deficiencia estructural para la generación de empleo decente o de calidad - con un contrato de trabajo, con seguridad social, con afiliación a una organización sindical y con ingresos iguales o mayores que el salario mínimo legal - debido a su estructura productiva poco diversificada y a la persistencia de una base rural importante con baja productividad económica. 
Esta baja productividad se explica en gran parte con los niveles de escolaridad que presentan los nicaragüenses y con la escaza infraestructura tecnológica en los procesos productivos de las micro y pequeñas empresas. Para el año 2009, según la EMNV (INIDE, 2009), las personas tenían en promedio seis años de escolaridad. La diferencia entre áreas de residencia es marcada ya que los nicaragüenses que viven en áreas rurales tienen niveles de escolaridad significativamente menores a los que viven en áreas urbanas: cuatro años contra siete años respectivamente.

\subsection{Contexto microeconómico}

Dado que las diferentes actividades laborales de los agentes económicos se agruparon en industrias según la similitud del trabajo que se realiza, es necesario apreciar quiénes integran cada una de las industrias. De esta manera se puede dar una imagen más detallada de las características específicas de cada industria en términos de subempleo.

Cuadro 1. Actividades que integran cada una de las industrias productivas

\begin{tabular}{|l|l|}
\hline \multicolumn{1}{|c|}{ Industria } & \multicolumn{1}{|c|}{ Actividades } \\
\hline Agrícola & $\begin{array}{l}\text { Agentes económicos que cultivan granos básicos, cereales y otros } \\
\text { tipos de cultivos. }\end{array}$ \\
\hline Pecuaria & $\begin{array}{l}\text { Agentes económicos que realizan actividades de cría de ganado } \\
\text { vacuno y cría de otros animales, así como agentes que elaboran } \\
\text { productos en base a la cría de animales. }\end{array}$ \\
\hline $\begin{array}{l}\text { Servicios } \\
\text { personales y } \\
\text { empresariales }\end{array}$ & $\begin{array}{l}\text { Agentes económicos inmersos en actividades de alquileres, } \\
\text { edición, consultorías, procesamiento de información, } \\
\text { impresiones, publicidad, lavandería, peluquería, limpia botas, } \\
\text { enseñanza, entre otros. }\end{array}$ \\
\hline Forestal & $\begin{array}{l}\text { Agentes económicos que realizan actividades de silvicultura, } \\
\text { extracción de madera y más. }\end{array}$ \\
\hline Pesquera & $\begin{array}{l}\text { Agentes económicos que realizan actividades de pesca y } \\
\text { acuicultura. }\end{array}$ \\
\hline Minera & $\begin{array}{l}\text { Agentes económicos que trabajan en extracción de minerales } \\
\text { metálicos, extracción de piedra, arena y arcilla, entre otros } \\
\text { minerales. }\end{array}$ \\
\hline Manufacturera & $\begin{array}{l}\text { Agentes económicos que laboran en actividades de transformación } \\
\text { o elaboración de productos diversos (leche, jabón, ropa, zapatos, } \\
\text { pintura, productos farmacéuticos, vidrio, aceite, pan). }\end{array}$ \\
\hline $\begin{array}{l}\text { Comercio, } \\
\text { hoteles y } \\
\text { restaurantes }\end{array}$ & $\begin{array}{l}\text { Agentes que se encuentran laborando en actividades de venta } \\
\text { "al por menor" y venta "al por mayor" de productos variados y en } \\
\text { actividades hoteleras. }\end{array}$ \\
\hline $\begin{array}{l}\text { Servicios } \\
\text { financieros y } \\
\text { conexos }\end{array}$ & $\begin{array}{l}\text { Agentes económicos que laboran en actividades de intermediación } \\
\text { financiera, en la autoridad monetaria, en actividades inmobiliarias } \\
\text { y en otras actividades monetarias (compra y venta de moneda). }\end{array}$ \\
\hline
\end{tabular}




\begin{tabular}{|l|l|}
\hline Transporte & $\begin{array}{l}\text { Agentes que trabajan en actividades de transporte de carga, } \\
\text { transporte regular de pasajeros, transporte aéreo, etc. }\end{array}$ \\
\hline Construcción & $\begin{array}{l}\text { Agentes que laboran en actividades de construcción de edificios/ } \\
\text { otros, en acondicionamiento de edificios y viviendas, y en } \\
\text { terminación de edificios y viviendas. }\end{array}$ \\
\hline Público & $\begin{array}{l}\text { Agentes económicos que laboran para el gobierno (médicos, } \\
\text { conductores oficiales, personal de limpieza, farmacología, } \\
\text { gerentes de empresas públicas, etc.) }\end{array}$ \\
\hline
\end{tabular}

Fuente: Con base en ECH (INIDE, 2010).

En el presente trabajo se entenderá como subempleo el deseo y disposición que tienen los agentes económicos (en cualquier industria) por trabajar más horas de las que laboraban al momento en que la encuesta fue aplicada. El hecho de que un individuo trabaje menos de 45 o más horas a la semana (8 horas de lunes a viernes más 5 horas sabatinas) y no desee laborar más tiempo no es considerado un subempleado, sino un inactivo puro.

Esta definición de subempleo que se utiliza no difiere sustancialmente de la definición propuesta por Ahijado (1985). Sin embargo, se considera más aplicable al contexto nicaragüense porque implica la imposibilidad del mercado de generar trabajo satisfactoriamente.

De esta manera, es oportuno conocer cuánto es el tiempo que dedican los agentes económicos a trabajar en la semana. Laborar menos de las 45 horas semanales no implica un problema per se. Cuando no existen deseos de trabajar más se asume que el individuo está satisfecho con su empleo, no obstante es evidente que no se está aprovechando a su totalidad la fuerza de trabajo. En definitiva, el factor productivo trabajo no es utilizado plenamente, significando así la renuncia a la producción de bienes y servicios que, de otra manera, pudieran haber sido producidos.

De los ocupados que no trabajan en organizaciones no gubernamentales y otras actividades no especificadas, se puede apreciar que es en el primer trimestre que los agentes económicos dedican más horas al trabajo (ver Cuadro 2). A pesar de esto no se logra alcanzar el tiempo completo que se debería trabajar a la semana y, de esta manera, se está perdiendo la oportunidad de producir más bienes y servicios. 
Encuentro No. 94, 42-61, 2013

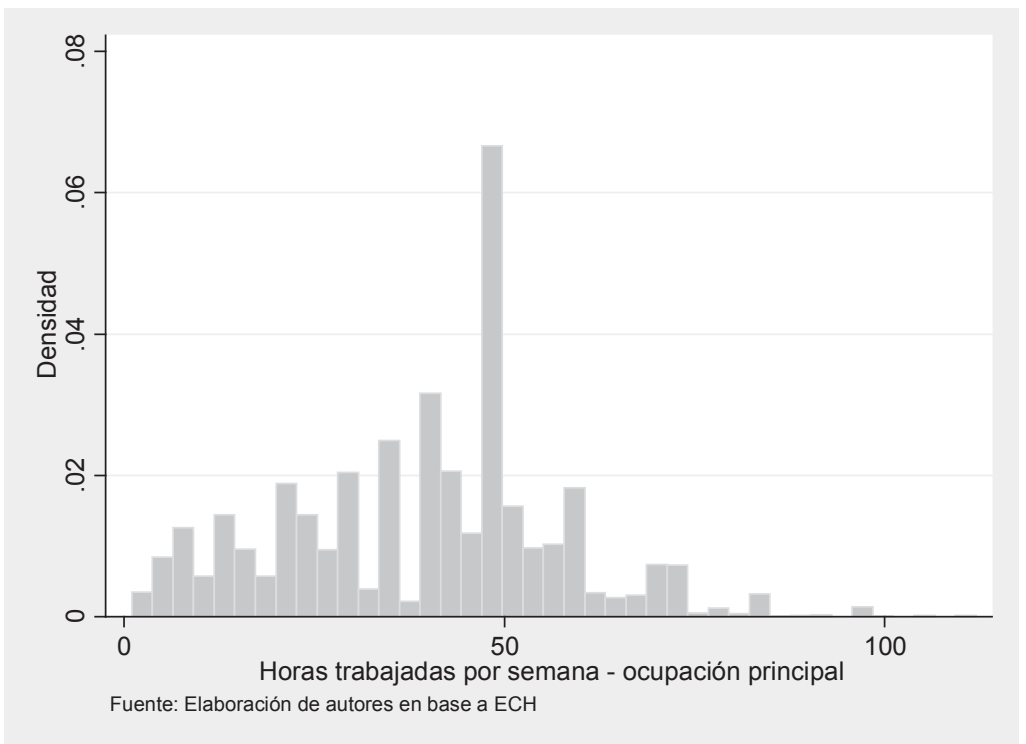

Ilustración 1. Distribución de horas trabajadas a la semana para los primeros tres meses del año.

Fuente: Elaboración de autores en base a ECH (INIDE, 2010)

El promedio utilizado puede alterar los resultados debido a que se está analizando una distribución de ingresos sesgada positivamente. En la primera fila del Cuadro 2 se toman en cuenta las observaciones que dedican más de 100 horas de trabajo a la semana. Esto tiende a alterar el promedio, sesgándolo hacia valores superiores, razón por la cual se quitan las observaciones más altas (con más de 70 horas semanales), obteniendo un promedio con un menor grado de sesgo.

En la Ilustración 1 se puede ver que, a pesar de la existencia de un gran número de personas que laboran alrededor de 45 horas a la semana para el primer trimestre del año, aún existe un gran segmento que labora muy por debajo de tales horas a la semana e incluso muy por encima de este valor, alcanzando hasta un máximo de 112 horas a la semana. Para los demás trimestres del año la distribución es muy similar a la presentada en la Ilustración 1.

Cuadro 2. Horas regularmente trabajadas a la semana

\begin{tabular}{|l|c|c|c|c|}
\hline \multicolumn{1}{|c|}{ Variable } & $\begin{array}{c}\text { ENERO, } \\
\text { FEBRERO, } \\
\text { MARZO }\end{array}$ & $\begin{array}{l}\text { ABRIL, } \\
\text { MAYO, } \\
\text { JUNIO }\end{array}$ & $\begin{array}{c}\text { JULIO, } \\
\text { AGOSTO, } \\
\text { SEPTIEMBRE }\end{array}$ & $\begin{array}{c}\text { OCTUBRE, } \\
\text { NOVIEMBRE, } \\
\text { DICIEMBRE }\end{array}$ \\
\hline $\begin{array}{l}\text { Horas } \\
\text { promedio } \\
\text { trabajadas } \\
\text { regularmente a } \\
\text { la semana }\end{array}$ & 39 & 37 & 37 & 37 \\
\hline
\end{tabular}




\begin{tabular}{|l|l|l|l|l|}
\hline $\begin{array}{l}\text { Horas } \\
\text { promedio } \\
\text { trabajadas } \\
\text { regularmente } \\
\text { para agentes } \\
\text { que laboran } \\
\text { menos de } \\
70 \text { horas a la } \\
\text { semana }\end{array}$ & 36 & 35 & 34 & 35 \\
\hline
\end{tabular}

Fuente: ECH (INIDE, 2010).

Sin embargo, esta imagen resulta aún muy superficial, por lo que se procedió a analizar el tiempo promedio (sin omisión de las observaciones que laboran más de 70 horas a la semana) dedicado al trabajo pero diferenciando por industrias productivas. Así se observó que la industria que presenta agentes económicos con mayor tiempo promedio de trabajo es la industria del transporte, seguida de los trabajadores en el sector público.

Esta característica del sector público como uno de los sectores que presenta un mayor número de personas trabajando en promedio más de 45 horas, puede comprenderse si se toma en cuenta que muchos de estos trabajadores pueden ser profesores, doctores, enfermeras, policías, soldados, bomberos, conserjes, etc., que laboran para entes gubernamentales.

En el caso de la industria del transporte puede significar el hecho de que las jornadas de transporte de carga pueden acumular largas horas, y es muy probable que los trabajadores inmersos en actividades de transporte sean trabajadores informales que sobrepasan las horas establecidas a la semana.

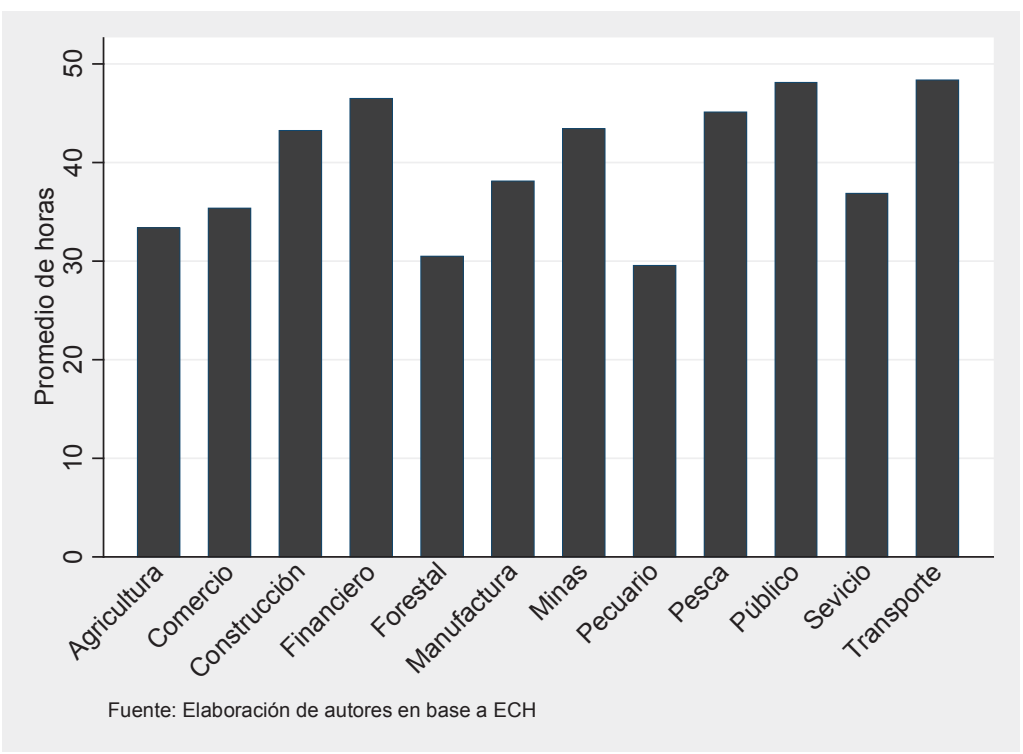

Ilustración 2. Horas promedio trabajadas por industria productiva

Fuente: Elaboración de autores en base a ECH (INIDE, 2010). 
Se puede observar que los agentes económicos involucrados en actividades agrícolas se encuentran dentro de una de las tres industrias con menos horas de trabajo a la semana en comparación a las demás. Los productores de granos básicos, hortalizas, legumbres y demás productos agroexportables trabajan en promedio 33.4 horas a la semana, y es en el primer trimestre donde los agricultores trabajan más horas (36 horas).

Esto puede ser explicado por el hecho de que Nicaragua es una economía eminentemente agropecuaria, con una marcada estacionalidad del desempeño productivo definida por el ciclo agrícola, en este caso, la cosecha de apante de granos básicos y la cosecha de primera de los principales productos agroexportables. Las actividades de agricultura, ganadería, silvicultura y pesca representan el 20\% del PIB anual y generan alrededor del 31\% del empleo (sin incluir el empleo generado en las actividades de comercio y de transporte que dependen de las actividades agropecuarias).

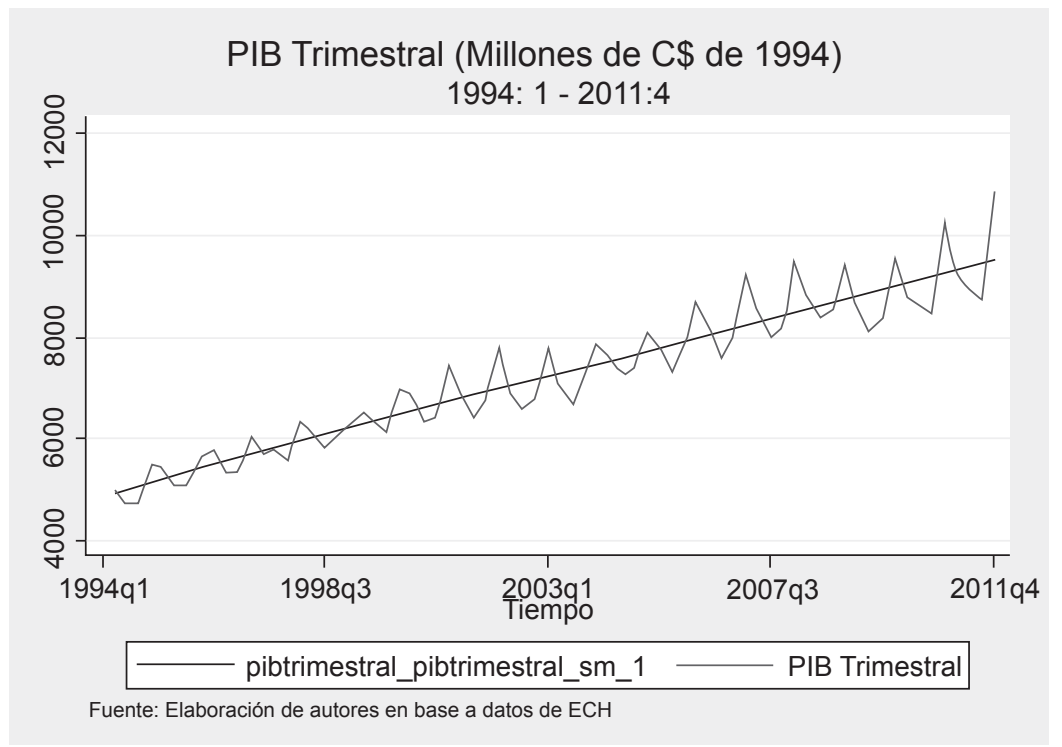

Ilustración 3. Estacionalidad del PIB real de Nicaragua

Fuente: Elaboración de autores en base a datos de ECH (INIDE, 2010).

Al ilustrar esta estacionalidad extrayendo los factores estacionales del PIB trimestral a precios de 1994 se observa que los trimestres de mayor desempeño productivo son el cuarto y el primero, en ese orden de importancia. A priori, habría que esperar que los problemas de subempleo sean más evidentes en el tramo comprendido entre el segundo y tercer trimestre del año.

Las industrias manufactureras, industrias de servicio e industrias de comercio, que aglomeran en conjunto un promedio de 52\% de los trabajadores de la economía - según estimaciones propias en base a la ECH (INIDE, 2010) - no se perfilan como industrias con bajas horas de trabajo a la semana pero no son industrias donde se trabaje plenamente las horas disponibles para producir. En términos simples, un individuo que se encuentra en alguna de estas tres industrias trabaja un promedio 
de siete horas y media al día de lunes a viernes, dejando el sábado libre y el domingo también.

En la teoría microeconómica se asume que las personas, al tener una dotación de tiempo a la semana, pueden decidir cuánto de éste se puede dedicar para trabajar o para hacer otro tipo de actividades comúnmente catalogadas como ocio.

Según Nicholson (2004), las horas de trabajo ofertadas son una función creciente de los salarios reales debido a que mayores salarios hacen que cada individuo en el mercado laboral trabaje más.

Consecuentemente, es oportuno apreciar los niveles de salarios que presenta cada industria. El salario, estimado como el ingreso proveniente de actividades laborales, se perfila como una de las variables que pueden influir en que un individuo se encuentre subempleado o no. Es de esperarse que si un agente económico cuenta con un nivel de salario alto no desee trabajar más horas, incluso si se trabaja menos de las 45 horas semanales.

Cuadro 3. Ingresos mensuales y coeficiente de Gini (promedios para los cuatro trimestres)

\begin{tabular}{|c|c|c|c|}
\hline \multirow{2}{*}{ Industria } & \multicolumn{2}{|c|}{ Salarios (ingresos) promedio } & \multirow{2}{*}{ Coeficiente de Gini } \\
\cline { 2 - 3 } & $\mathrm{C} \$$ de 2010 & US\$ de 2010* & \\
\hline Agricultura & 2,367 & 111 & 44.8 \\
\hline Pecuario & 2,974 & 139 & 42.3 \\
\hline Servicio & 5,984 & 280 & 54.0 \\
\hline Forestal & 3,535 & 166 & 48.0 \\
\hline Pesca & 5,362 & 251 & 38.9 \\
\hline Minas & 6,967 & 326 & 56.0 \\
\hline Manufactura & 5,622 & 263 & 48.2 \\
\hline Comercio & 4,025 & 188 & 52.8 \\
\hline Transporte & 6,671 & 312 & 50.3 \\
\hline Financiero & 12,515 & 586 & 39.5 \\
\hline Construcción & 4,698 & 220 & 51.0 \\
\hline Público & 12,215 & 572 & 28.0 \\
\hline
\end{tabular}

${ }^{*}$ De acuerdo con el tipo de cambio oficial promedio de C\$23.55 x US\$ 1 en 2010. Fuente: Elaboración de autores en base a ECH (INIDE, 2010).

El Cuadro 3 presenta una característica preocupante de la realidad económica de Nicaragua: si se parte del hecho de que las industrias agropecuarias son las industrias que sostienen la economía nacional en términos del empleo que aglomeran y en términos de la composición de los productos de exportación con los que contribuyen, se puede observar que son éstas las industrias que presentan los más bajos niveles de ingreso promedio (para la industria pecuaria se estima un ingreso promedio de $\mathrm{C} \$ 2,974$ y para la industria agrícola se estima un ingreso promedio de C\$2,367 en 2010). Si se asume que los salarios se igualan a la productividad marginal, entonces queda en evidencia que las industrias agropecuarias no presentan altos niveles de productividad. 
Estas características que presenta la economía nicaragüense se agudizan si se observa que ambas industrias, pecuaria y agrícola, son dos de los tres sectores que dedican menos tiempo a laborar. Dicho esto, se tienen industrias que no generan mucho valor agregado y se tienen industrias que laboran muy poco tiempo en relación con las demás industrias del país, dejando así un trecho de bienes y servicios que se pudieran producir de mejor manera y en mayores cantidades.

En el Cuadro 3 se constata que la industria financiera es la que presenta mayores niveles de ingreso. Los agentes económicos que trabajan en actividades relacionadas con intermediación financiera y demás actividades similares ganan en promedio 5.28 veces más que los agricultores. Esta desigualdad, que no sólo se da en dicha industria, también se demuestra con un coeficiente promedio de Gini de 46.12 para 12 de las industrias en todos los trimestres del año según estimaciones en base de la ECH 2010 (INIDE, 2010).

Los individuos que trabajan en tareas de transporte urbano colectivo, o proveyendo servicios de transporte privado o de carga, son los que trabajan más, pero no son los que tienen mayores niveles de ingreso salarial.

\subsection{Estimación de modelo econométrico}

\subsubsection{Datos}

Para la realización del modelo econométrico se procedió a limpiar la base de datos de la ECH (INIDE, 2010). Las actividades de los individuos que tienen similitud se agrupan en las industrias presentadas en el Cuadro 5 y se realizó el mismo procedimiento para cada trimestre que permite la ECH 2010. A continuación se presenta el número de observaciones promedio de los cuatro trimestres para cada una de las variables estimadas.

Cuadro 5. Datos a utilizar en el modelo econométrico

\begin{tabular}{|c|c|c|c|}
\hline Industrias & $\begin{array}{c}\text { Observaciones } \\
\text { promedio estimadas } \\
\text { de los 4 trimestres }\end{array}$ & $\begin{array}{c}\text { Peso promedio de los } \\
\text { 4 trimestres (\%) }\end{array}$ & Variable \\
\hline Agricultura & $4,395.8$ & 32.5 & Dicótoma \\
\hline Pecuario & 477.8 & 3.5 & Dicótoma \\
\hline Servicio & $1,704.0$ & 12.6 & Dicótoma \\
\hline Forestal & 68.0 & 0.5 & Dicótoma \\
\hline Pesca & 85.0 & 0.6 & Dicótoma \\
\hline Minas & 44.8 & 0.3 & Dicótoma \\
\hline Manufactura & $1,714.8$ & 12.7 & Dicótoma \\
\hline Comercio & $3,627.8$ & 26.9 & Dicótoma \\
\hline Transporte & 534.8 & 4.0 & Dicótoma \\
\hline Financiero & 136.5 & 1.0 & Dicótoma \\
\hline Construcción & 565.0 & 4.2 & Dicótoma \\
\hline
\end{tabular}




\begin{tabular}{|c|c|c|c|}
\hline Público & 140.5 & 1.0 & Dicótoma \\
\hline Variable & $\begin{array}{c}\text { Observaciones } \\
\text { promedio estimadas } \\
\text { de los 4 trimestres }\end{array}$ & $\begin{array}{c}\text { Promedio de los 4 } \\
\text { trimestres }\end{array}$ & Variable \\
\hline Ingresos & $10,055.5$ & $5,085.5$ córdobas & Continua \\
\hline Educación & $17,200.3$ & 5.92 años & Continua \\
\hline $\begin{array}{c}\text { Variable } \\
\text { dependiente }\end{array}$ & $\mathrm{Y}=1$ Agente desea trabajar más horas $\mid \mathrm{Y}=0$ Agente no desea \\
trabajar más horas
\end{tabular}

Fuente: Estimación de autores en base a ECH (INIDE, 2010).

Las variables utilizadas para la estimación econométrica son las presentadas en el Cuadro 5, donde la mayoría son variables dicótomas (dummies) que toman el valor " 1 " si la observación se encuentra en la industria que indica el nombre de la variable y "0" si es lo contrario.

Adicionalmente se estimaron dos variables continuas: la variable Ingresos representa el valor del ingreso generado por actividades relacionadas al trabajo, y la variable llamada Educación representa los años de educación con los que cuentan los individuos.

Para la estimación de los años de educación - de acuerdo con la ECH 2010 - se establecieron los siguientes supuestos: Técnico básico - 8 años ya estudiados; Técnico medio - 9 años ya estudiados; Formación docente - 9 años ya estudiados; Técnico superior - 12 años ya estudiados; Universitario - 12 años ya estudiados; Maestría - 17 años ya estudiados; Doctorado - 19 años ya estudiados. Se asume que los años de escolaridad empiezan a contabilizarse desde el primer grado de primaria.

\subsubsection{Estimación}

Se estima un modelo Logit en donde se establece la siguiente forma funcional:

$$
\text { (3) } \quad p=\frac{1}{1+e^{-Z}}
$$

Donde $Z=\alpha+\beta 1 *$ Agricultura $+\beta 2 *$ Servicio $+\beta 3 *$ Manufactura + $\beta 4 *$ Comercio $+\beta 5 *$ Construcción $+\beta 6 *$ Ln (Ingresos $)+\beta 7 *$ Educación $+u$

Donde es el parámetro a calcular para cada una de las variables independientes para i: $1,2,3,4 \ldots . . n$ y es el valor de la constante y $u$ un término de error.

La variable dependiente equivale a 1 si el agente económico desea trabajar más, está dispuesto y no puede (subempleado) o equivale a 0 si es lo contrario. 
Cuadro 6. Estimaciones de los efectos marginales del modelo logístico

\begin{tabular}{|c|c|c|c|c|}
\hline Variable & $\begin{array}{l}\text { Primer } \\
\text { trimestre }\end{array}$ & $\begin{array}{l}\text { Segundo } \\
\text { trimestre }\end{array}$ & $\begin{array}{c}\text { Tercer } \\
\text { trimestre } \\
\end{array}$ & $\begin{array}{l}\text { Cuarto } \\
\text { trimestre }\end{array}$ \\
\hline \multicolumn{5}{|c|}{ Efectos marginales - evaluados en las medias } \\
\hline Agricultura & $-0.076^{* * *}$ & 0.027 & 0.03 & 0.029 \\
\hline Error estándar & 0.018 & 0.023 & 0.023 & 0.022 \\
\hline Servicio & $0.12^{* * *}$ & $0.091^{* * *}$ & $0.104^{* * *}$ & $0.076^{* * *}$ \\
\hline Error estándar & 0.02162 & 0.022 & 0.021 & 0.021 \\
\hline Manufactura & $0.089^{* * *}$ & $0.059^{* * *}$ & $0.101^{* * *}$ & $0.068^{* * *}$ \\
\hline Error estándar & 0.02179 & 0.022 & 0.021 & 0.021 \\
\hline Comercio & $0.05^{* * *}$ & $0.041^{* *}$ & $0.07^{* * *}$ & $0.0728^{* * *}$ \\
\hline Error estándar & 0.01871 & 0.02 & 0.019 & 0.0194 \\
\hline Construcción & $0.082^{* * *}$ & $0.16^{* * *}$ & $0.137^{* * *}$ & $0.154^{* * *}$ \\
\hline Error estándar & 0.02792 & 0.027 & 0.028 & 0.028 \\
\hline Ln (ingresos) & $-0.12^{* * *}$ & $-0.18^{* * *}$ & $-0.17^{* * *}$ & $-0.14^{* * *}$ \\
\hline Error estándar & 0.005 & 0.006 & 0.006 & 0.005 \\
\hline Educación & $0.004^{* * *}$ & $0.004^{* * *}$ & $0.004^{* * *}$ & $0.003^{* * *}$ \\
\hline Error estándar & 0.00118 & 0.001 & 0.0013 & 0.0013 \\
\hline Pseudo R`2 & 0.084 & 0.11 & 0.09 & 0.079 \\
\hline Log Liklihood & -5083.3257 & -5069.5923 & -5301.2017 & -5547.1137 \\
\hline LR chi2(7) & 941.23 & 1332.79 & 1176.17 & 957.59 \\
\hline Prob > chi2 & 0.000 & 0.000 & 0.000 & 0.000 \\
\hline Observaciones & 8984 & 8418 & 8531 & 8858 \\
\hline $\begin{array}{l}\text { Proporción de } \\
\text { observaciones } \\
\text { predichas } \\
\text { correctamente }\end{array}$ & $71.50 \%$ & $68.21 \%$ & $66.70 \%$ & $65.70 \%$ \\
\hline $\begin{array}{l}{ }^{*} \text { Significativo al } \\
90 \%\end{array}$ & \multicolumn{2}{|c|}{ **Significativo al 95\% } & icativo al 99\% & \\
\hline
\end{tabular}

Fuente: Estimación de autores en base a ECH (INIDE, 2010).

Tomando en cuenta que la mayoría de las variables independientes son variables dicótomas, se debe de mantener un grupo base con el cual hacer una comparación (benchmark). El grupo base considerado para el modelo incluye las siguientes industrias: pública, forestal, minera, pecuaria, financiera, transporte. De esta manera, la interpretación de los efectos marginales se hace con relación al grupo base definido.

Junto a las estimaciones de los efectos marginales del modelo Logit se presenta la validación del mismo mediante la prueba Pearson. 
Cuadro 7. Validación de modelo Logit. Prueba Pearson.

\begin{tabular}{|l|l|l|l|l|}
\hline Prueba Pearson & Primer trimestre & Segundo trimestre & Tercer trimestre & Cuarto trimestre \\
\hline Ho: El modelo está bien ajustado & $\begin{array}{l}\text { Se acepta Ho } \\
(* * *) \text { Prob }>\text { Chi2 }=0.74\end{array}$ & $\begin{array}{l}\text { Se acepta Ho } \quad(* * *) \\
\text { Prob }>\text { chi2 }=0.1810\end{array}$ & $\begin{array}{l}\text { Se acepta Ho }(* * *) \\
\text { Prob }>\text { chi2 }=0.034\end{array}$ & $\begin{array}{l}\text { Se acepta Ho } \\
(* * *) \\
\text { Prob }>\text { chi2 }=0.151\end{array}$ \\
\cline { 1 - 1 } Hi: Falta ajuste en el modelo & & & &
\end{tabular}

Fuente: Estimación de autores en base a datos de ECH (INIDE, 2010).

Una vez que se validó el modelo econométrico se procedió a interpretar los resultados. En primer lugar, es necesario señalar que la industria agrícola es estadísticamente diferente de cero únicamente en el primer trimestre del año. Para los siguientes trimestres del 2010 se pierde significancia a pesar de presentar el signo esperado. Sin embargo, el modelo econométrico en general mantiene su buen ajuste en todos los trimestres incluso al 99\% de significancia.

Según los parámetros estimados, que representan los efectos marginales evaluados en las medias de las variables independientes, los individuos que se encuentran trabajando en actividades agrícolas tienen 7.6 puntos porcentuales de menores de probabilidades de desear trabajar más horas a la semana con respecto a trabajadores en otros sectores (grupo base). A su vez, el signo negativo de la industria agrícola, para el primer trimestre del 2010, es congruente con la estacionalidad del Índice Mensual de la Actividad Económica para esta actividad, que indica que en conjunto en los tres primeros meses del año, el desempeño productivo de esta actividad se encuentra por encima del desempeño promedio anual.

En este sentido, se puede asociar la pérdida de significancia estadística que presenta la industria agrícola en los siguientes tres trimestres del año con la marcada estacionalidad de su ciclo productivo.

En el primer trimestre del año un trabajador en la industria de servicios tiene 12 puntos porcentuales de mayores probabilidades de estar subempleado en relación con otros sectores representados en el grupo base. Para los demás trimestres, ésta probabilidad oscila en un rango de 7.6 a 10.4 puntos porcentuales.

Para la industria manufacturera, la mayor probabilidad de presentar trabajadores subempleados con respecto a otros sectores se encuentra en el tercer trimestre del año (10 puntos porcentuales de mayores probabilidades con respecto a los demás sectores).

Para la actividad comercial se presentan probabilidades positivas de estar subempleado muy similares a lo largo del año 2010. Éstas varían en un rango de 4.0 y 7.2 puntos porcentuales con respecto a las demás industrias, lo cual implica que las personas que laboran en actividades comerciales tienen más probabilidades de querer trabajar más y no poder en relación al grupo base.

Según los resultados obtenidos, la industria de la construcción es la industria con mayor probabilidad de generar puestos de trabajo en condiciones de subempleo. Esto es un indicador de insatisfacción en las labores de esta actividad. En promedio, los individuos que trabajan en actividades de construcción tienen 13 puntos porcentuales de mayores probabilidades de querer trabajar más - estar dispuesto a hacerlo y no poder - si se compara con las industrias del grupo base.

Enfocando el análisis a la primera de las variables continuas ( $\mathrm{Ln}^{*}$ Ingresos) se encuentra que los signos estimados concuerdan con los signos esperados porque a 
mayores niveles de ingresos los trabajadores tienden a tener menos probabilidades de querer trabajar más horas. El coeficiente estimado indica que ante aumentos en el logaritmo natural de los salarios derivados de actividades económicas se tiene, en promedio, 15 puntos porcentuales de menores probabilidades de querer trabajar más horas a la semana para cualquier sector de la economía, es decir, sin hacer distinción entre industrias.

Por otro lado, el signo del coeficiente estimado para la variable educación indica que, con un año más de educación, la probabilidad de desear trabajar más horas a la semana aumenta en 0.4 puntos porcentuales. Este es un resultado muy interesante pues evidencia el bajo nivel de escolaridad en la economía. Así mismo, este signo y baja magnitud del parámetro de educación es el reflejo de la incapacidad que tiene el mercado de generar suficientes y adecuadas plazas de trabajo.

\section{Conclusiones y recomendaciones}

En el presente artículo se hizo uso de la Encuesta Continua de Hogares (INIDE, 2010) para analizar una temática no muy explotada a nivel de la literatura económica en Nicaragua, que es el subempleo a nivel de las industrias productivas del país.

Los resultados de las estimaciones indican que todas las industrias presentan trabajadores subempleados. La industria que presenta una mayor probabilidad de generar puestos de trabajo en condiciones de subempleo es la industria de la construcción, seguida por la industria de servicios y manufactura. Esta característica del mercado laboral nicaragüense implica la existencia de insatisfacción o inconformidad laboral.

Las industrias que presentan menores probabilidades de generar puestos de trabajo en condiciones de subempleo son las industrias agrícolas - aunque se pierde significancia estadística para los tres últimos trimestres del año de estudio - y las industrias comerciales. En ambas actividades se confirma, pues, el predominio del desempleo abierto sobre el subempleo.

Así mismo, se evidencia que individuos con mayores niveles salariales tienen menos probabilidades de desear trabajar más horas a la semana, independientemente de la industria productiva en la que se encuentren.

Los resultados obtenidos para la variable educación, si bien no presentan una magnitud muy alta, reflejan una problemática de la economía nacional en relación con los bajos niveles de escolaridad imperantes. El hecho de que a mayores niveles educativos se tengan mayores probabilidades de desear trabajar más también puede significar la incapacidad del mercado de generar puestos de trabajo adecuados a mayores niveles de calificación.

Finalmente se presentan algunas recomendaciones de políticas públicas dirigidas a combatir el subempleo:

- La formulación de políticas de empleo con la participación del gobierno, los sindicatos y los gremios de productores.

- La promoción de educación técnica media y superior para elevar la productividad laboral. 
- $\quad$ El apoyo al desarrollo de las MYPIMES.

- El fortalecimiento del Banco de Fomento "Produzcamos", con el fin de transferir crédito y tecnología a un bajo costo.

- Legislar para la igualdad salarial de hombres y mujeres en el mismo puesto de trabajo.

- Reducir los trámites burocráticos para la creación de empresas.

\section{Agradecimientos}

Se agradecen las valiosas enseñanzas de Carlos Narváez Silva, profesor de la Facultad de Ciencias Económicas y Empresariales de la Universidad Centroamericana (UCA)

\section{Anexos}

Se presentan las funciones de distribución acumulativas para los modelos estimados referentes a los trimestres i, ii, iii, y iv. Se presenta que los efectos marginales de las variables explicativas no son constantes. El efecto parcial es mayor cuando xbeta se iguala a cero y más pequeño a medida que xbeta tiende a menos infinito y a más infinito.

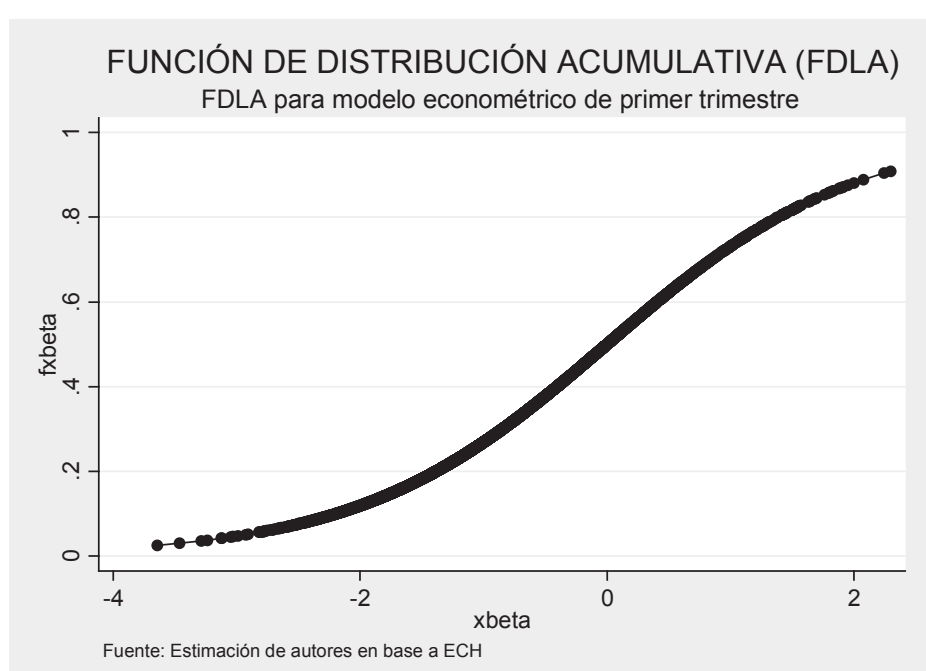


Encuentro №. 94, 42-61, 2013

\section{FUNCIÓN DE DISTRIBUCIÓN ACUMULATIVA (FDLA)}

FDLA para modelo econométrico de segundo trimestre

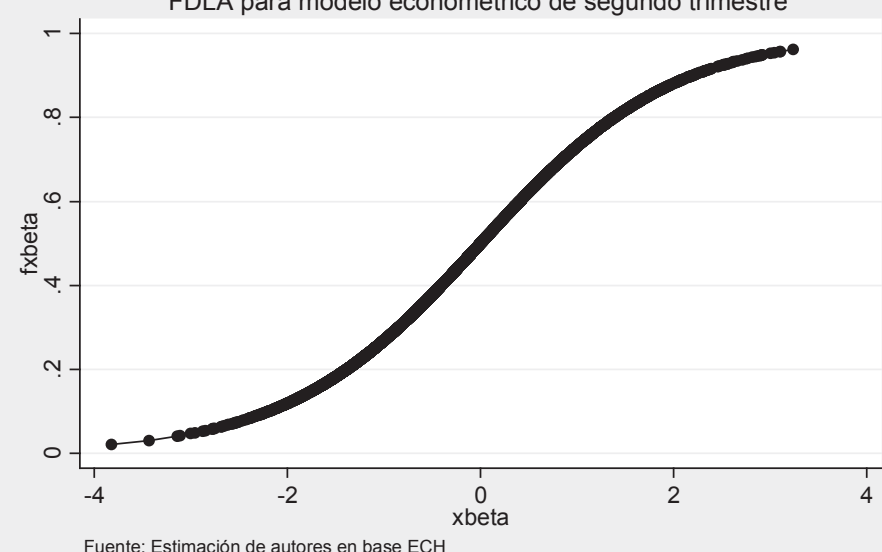

FUNCIÓN DE DISTRIBUCIÓN ACUMULATIVA (FDLA)

FDLA para modelo econométrico de tercer trimestre

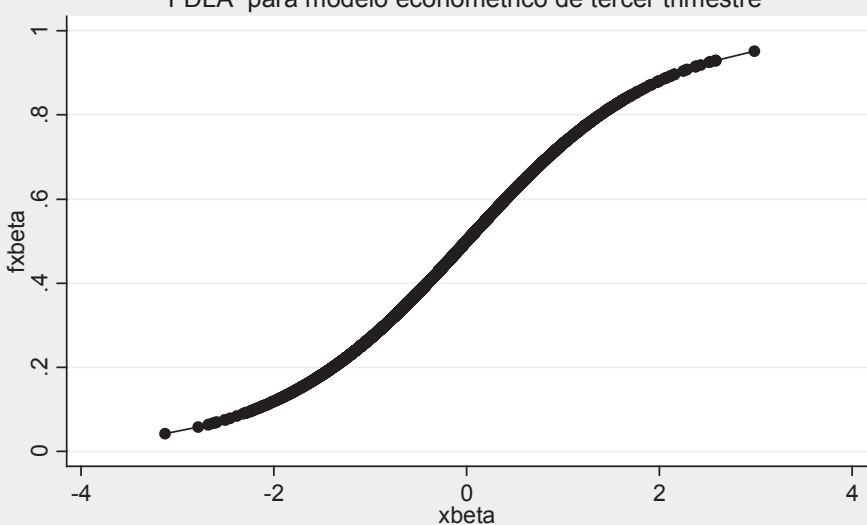

Fuente: Elaboración de autores en base a ECH

FUNCIÓN DE DISTRIBUCIÓN ACUMULATIVA (FDLA)

FDLA para modelo de cuarto trimestre

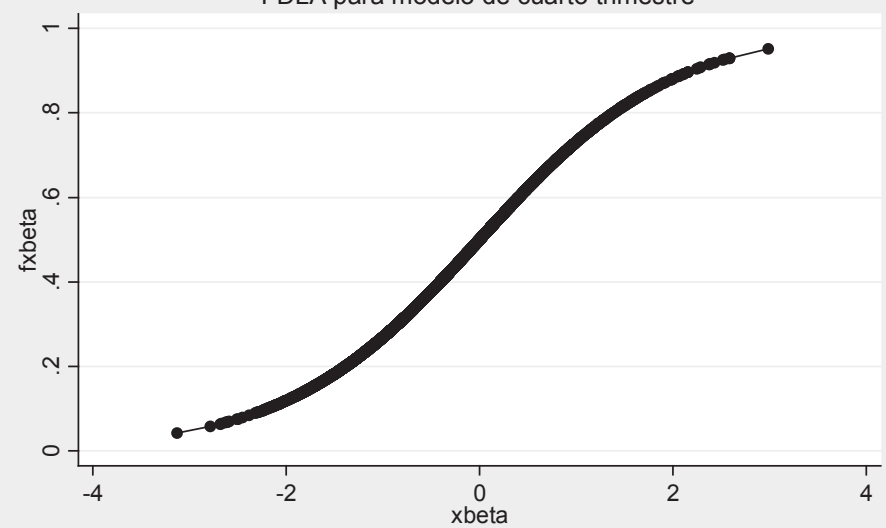

Fuente: Elaboración de autores en base a ECH 


\section{Referencias bibliográficas}

Ahijado, M. (1985). Diccionario de Teoría Económica. Madrid: Pirámide.

Banco Central de Nicaragua. [BCN]. (1994). Sistema de Cuentas Nacionales de Nicaragua. Año base 1994.Recuperado el 10 de mayo de 2012, de http://www. bcn.gob.ni/publicaciones/eventuales/metodologias/Metodologia_SCN_ Nicaragua_base_1994.PDF

Banco Central de Nicaragua. (2011). Anuario de Estadísticas Mensuales. Recuperado el 20 de mayo de 2012, de http://www.bcn.gob.ni.

Bernal, R. (2008). The Informal Labor Market in Colombia: Identification and Characterization. Centro de Estudios de Desarrollo Económico: Facultad de Economía y CEDE . Recuperado el 12 de mayo de 2012, de http://www. undp.org.ni/

Gamboa, M. (2007). Flexibilización del mercado laboral en Nicaragua. Managua: Programa de Naciones Unidas para el Desarrollo.

Instituto Nacional de Información de Desarrollo [INIDE]. (2009). Encuesta de Medición del Nivel de Vida-EMNV. Recuperado el 10 de mayo de 2012, de http://www.inide.gob.ni/.

Instituto Nacional de Información de Desarrollo [INIDE]. (2010). Encuesta Continua de Hogares (ECH) Recuperado el 15 de mayo de 2012, de http://www.inide. gob.ni.

Bell, N. F. \& Blanchflower, D. (2011). Youth Underemployment in the UK in the Great Recession. New Hampshire: Universidad de Dartmouth. Recuperado el 5 de mayo de 2012, de www.dartmouth.edu

Nicholson, W. (2004). Teoría Microeconómica Principios Básicos y Ampliaciones. Cincinnati: Thomson.

North, D. (1990). Institutional Change and Economic Performance. Cambridge: Cambridge University Press. 